\title{
Exploring Small-World-Like Topologies Via SplitProber: Turning Power Laws into an Advantage in Unstructured Overlays
}

\author{
Xinli Huang, Wenju Zhang, Fanyuan Ma, and Yin Li \\ Department of Computer Science and Engineering, \\ Shanghai Jiao Tong University, Shanghai, 200030, P.R. China \\ \{huang-xl, zwj03, fyma, li-yin\}@sjtu.edu.cn
}

\begin{abstract}
Recent unstructured Peer-to-Peer systems, represented by Gnutella and Freenet, offer an administration-free and fault-tolerant application-level overlay network. While elegant from a theoretical perspective, these systems have some serious disadvantages. First, due to knowing very little about the nature of the network topology, the search algorithms operating on these networks result in fatal scaling problems. Second, these systems rely on application-level routing, which may be inefficient with respect to network delays and bandwidth consumption. In this paper, we propose a novel search algorithm, called SplitProber, to explore the small-world-like topologies of these networks efficiently and scalablely, by turning the power-law degree distributions in these networks to an advantage, and by making discriminative use of nodes according to their different roles in the network. As a result, we are able to reconcile the conflict of remedying the mismatch between the overlay topology and its projection on the underlying physical network, while at the same time navigating these networks with a guaranteed high efficiency and using only local knowledge as cues. Our simulation results indicate that the proposed algorithm outperforms several other well-known methods with significant performance gains.
\end{abstract}

\section{Introduction}

Providing large-scale and efficient content storage and delivery are becoming ever so important because the demand for Internet-based applications is growing at an incredible rate. Recent application-level unstructured overlay networks, such as Gnutella [1] and Freenet [2], due to their abilities to support uncoupled data allocation, complex semantic queries, self-organization in highly dynamic scenario, are considered more competent for these Internet-based applications, compared with structured overlays where both the data placement and the overlay topology are tightly controlled, such as CAN [3], Chord [4], and Pastry [5]. Nodes in these networks interact symmetrically and collectively contribute towards an administrationfree and fault-tolerance decentralized storage space, by hiding the underlying dynamism and heterogeneity in these systems. While elegant from a theoretical perspective, these systems suffer from two limitations. First, due to knowing very little about the nature of network topology, the search algorithms operating on these networks, as 
in the case of Gnutella, conduct a "blind" and explosive broadcast search, which results in fatal scaling problems. Second, these systems rely on application-level routing (search) that largely ignores the characteristics of the underlying physical networks, which leads to an unnecessarily large network delays with very high bandwidth consumption. We argue that for a system to function efficiently, it is important for the search algorithm to take into account both the intrinsic topological nature stemmed in this system and its projection on the underlying network.

To illustrate the topological nature behind these unstructured overlays, recent studies in measurements of Gnutella [6] and simulated Freenet networks [7], show that they contain a few nodes that have a very high degree and many with low degree following power-law distributions, that is, the probability that a node has $k$ links is approximately proportional to $1 / k^{\tau}$, where $\tau$ is the scaling exponent. This power law in the link distribution reflects the presence of central individuals who interact with many others and play a key role in relaying information, a fact that can be exploited when designing efficient search algorithms. In addition, further study of [6] shows that Gnutella topologies demonstrate strong "small-world" phenomena: characteristic path length is comparable to that of a random graph, while the clustering coefficient stays at a very high level similar to that of a regular graph. The small-world phenomenon is pervasive in networks arising from society, nature and technology. In many such networks, empirical observations suggest that any two individuals in the network are likely to be connected through a short sequence of intermediate acquaintances $[8,9]$. One network construction that gives rise to small-world behavior is where each node in the network knows its physical neighbors, as well as a small number of randomly chosen distant nodes. The latter represent shortcuts in the network. It has been shown that this construction leads to graphs with small diameter, leading to a small routing distance between any two individuals $[9,10]$.

The power-law degree distributions and small-world phenomena in Gnutella-like unstructured overlays can significantly impact the performance of algorithms such as those for routing or searching [9]. Therefore the existence of these properties in these networks presents an important issue to consider when designing new, more scalable application-level algorithms and protocols. As is pointed out in [11], the presence of high degree nodes in a power-law graph, so useful to speed up the search process, ironically, also worsens the search cost scaling with problems of traffic explosion and hotspots, which results in poor utilization of network bandwidth and hinders scaling. In this sense, the question we try to answer in this paper is, whether the search algorithms operating on such overlays can be coupled with considerations of these intrinsic topological nature and conduct the search process in a fashion of relatively high and but also balanced search efficiency and network utilization, using only local knowledge.

In this paper, we propose a novel search algorithm, called SplitProber, to explore these networks efficiently and scalablely, by turning the power-law degree distributions in these networks to an advantage, and by making discriminative use of nodes according to their different roles in the network. As a result, we are able to reconcile the conflict of compensating the mismatch between the overlay topology and its projection on the underlying physical network, while at the same time navigating these networks with a guaranteed high efficiency and using only local knowledge as cues. 
The main contributions of this paper are: (a) we develop a provably effective model for building unstructured overlays with desirable topological properties; (b) Based on this model, we investigate several useful techniques to realize the efficient local search in these overlay topologies and propose SplitProber, a novel local search algorithm by better exploiting the power-law degree distributions and the smallworld-like structure; (c) To further improve the performance of SplitProber, we also devise several unique optional optimization mechanisms as enhancements.

The remainder of the paper is organized as follows. Section 2 provides related work. Section 3 discusses the design of the SplitProber algorithm in detail. We provide the evaluation methodology in Section 4 and then present our analytical simulation results in Section 5. In the last section, we conclude this paper and highlight some directions as future work.

\section{Related Work}

All the above facts indicates that a realistic link-distribution plays a crucial role in the effectiveness of the search strategy and that it might be a crucial ingredient in explaining the low diameter seen in messaging passing on the small-world-like unstructured overlays. To navigate these overlays efficiently, several alternative approaches have been proposed in the near past to examine the role of link distribution in overlay topology, and to utilize it for the performance optimization.

Motivated by real experiments with social networks, Kleinberg was concerned with how, given the fact that short paths existed, one could find them without complete global information. The treatment given in $[9,12]$ had an elegant result, but the underlying graph model did not reflect all of the important features real world problems. An important shortcoming is its particular assumption of an inverse square correlation which implies that a majority of ones contacts lie in geographical proximity. What happens if a large fraction of people know as many people outside of their city or state as inside? Would it become impossible to pass messages efficiently?

Adamic et al in [11] prefer switching from broadcasting queries to passing them only to high-degree nodes, a situation similar to that of supernodes in the FastTrack network [13]. They assumed that higher-degree peers are also capable of higher query throughputs. However without some balancing design rule, such peers would be swamped with the entire P2P signaling traffic.

The fact that the number of hops between nodes is shorter in a power-law graph implies that the broadcasting method of locating nodes and resources will return results more quickly, which inspires Yang and Garcia [14] to propose a method called iterative deepening. The method is an improvement over the default protocol when the queries can be satisfied by nodes closer than the maximum radius defined by the TTL of the default. In that case, bandwidth and processing cost are saved. Similarly to some extent, Lv et al in [15] argue that by making better use of the more powerful peers, Gnutella's scalability issues could be alleviated. Instead of its flooding mechanism, they used random walks. Their preliminary design to bias random walks towards high capacity nodes did not go as far as the ultra-peer proposals in that the indexes did not move to the high capacity nodes. 
The above approaches have examined strategies for finding a node on a network knowing nothing other than the identities of one's first and second neighbors. However, a node can learn about the network over time and adapt its search strategies. Based on this intuition, a class of algorithms called adaptive search, represented by [16] and [17], suggests that adapting the search algorithm to incorporate information learned about the network can deliver results comparable to BFS (broadcast) search while using considerably less processing power and bandwidth. However, such topological changes might destroy the merits of the power-law degree distribution and its resultant small-world characteristics.

In summary, these Gnutella-related investigations are characterized by a bias for high degree peers and very short directed query paths, a disdain for flooding, and concern about excessive load on the "better" peers. Generally, the analysis and utilization of both the overlay nature and its mapping on the physical level remains open.

\section{SplitProber: Model and Algorithm}

In this section, we detail the model we use to build desirable overlays and the main ideas of the SplitProber algorithm, including several optimization mechanisms.

As is shown analytically in [11], with high-degree seeking local search strategies in power-law graphs, the length of the average path found grows slowly as the size of the network increases, whereas the average cost in the amount of time necessary to find the path scales nearly linearly. The extremely high cost of this procedure suggests that additional clues as to the location of the target or knowledge from the network over time would be necessary to make such an approach worthwhile.

We seek the advantages of power-law degree distributions and design overlays with a small-world structure. In our model of overlay topologies, a node's links to its neighbors are divided into two categories: local links and global links. The local links connect close nodes and the global links connect nodes chosen randomly. The fraction of links that are local, called the proximity factor $(\delta)$, is a key design parameter that controls the properties of the resultant overlay topology. Different values of $\delta$ let us span the spectrum of this class of overlay topologies.

To examine the role of local links on the impact of the average search time, we give a rough theoretical complexity analysis below. If the expected degree of a neighbor is $D$ and the fraction of random links is $(1-\delta)$ according to the above definition of the proximity factor, then it would have approximately $D^{2}$ second neighbors out of which $(1-\delta)$ would be long range or random links so the average time it would take to reach the target if it were to never use local links is:

$$
\mathrm{T}_{\text {global only }}=\frac{N}{D^{2}(1-\delta)}+\beta
$$

where $\beta$ is the average minimum number of steps required to reach the target.

The maximum number of steps it takes if the walker starts using local links after reaching within a distance $l$ of the target will be following:

$$
\mathrm{T}_{\text {global+local }}=\frac{N}{D^{2} l(1-\delta)}+\frac{\alpha l}{\delta}+\beta
$$


where $\alpha$ and $\beta$ are constants. For a power law graph of power approximately 2 as is observed in Gnutella networks [18], we have the expression for $D$ in terms of $N$ :

$$
D=\frac{N^{1 / 2}}{\log (N)}
$$

Now, substituting (3) into (2) and minimizing the resulting expression with respect to $l$ we get an upper bound for the average number of steps:

$$
\mathrm{T}_{\text {global+local }}=\sqrt{\frac{\alpha}{\delta(1-\delta)}} \log (N)+\beta
$$

According to the results of (1) and (4), it is obvious that the most efficient search is when there are both local and random long distance links. On getting close to the target say a distance $l$, it becomes advantageous to use local as opposed to globally random links. This kind of topology is expected to allow one to search the power-law graph more rapidly using only local knowledge. Then here comes an important issue: how to navigate the topology in the mentioned fashion efficiently? In our proposed SplitProber algorithm below, we introduce novel techniques which operate independently and in a decentralized manner to achieve this goal.

To turn power-law degree distribution into an advantage while at the same time minimizing the search cost scaling mentioned in Section 2, we advocate directing queries towards deliberately selected high-degree neighbors with the probability of a node being chosen proportional to its degree, following $P\left(N_{i}\right) \sim\left(\operatorname{Degree}\left(N_{i}\right)\right)^{\kappa}$, where $\kappa$ is defined as the selection strength imposed upon the high-degree nodes. Given the dynamic conditions of both the peer content and its location in a peer-to-peer network, we devise a unique adaptive better-neighbor selection scheme, by effectively coupling the "best results" scheme with the "best physical proximity" scheme. A SplitProber node first caches the nodes which had previously delivered a specified number of results in the least amount of time into its candidate neighbors list, and then updates its neighborhood by periodically evaluating the "distance" to the nodes in this list and its neighborhood according to a novel technique called Landmark Clustering [19] explained below.

Landmark Clustering. We pick $m$ landmark nodes that are randomly scattered in the overlay. These landmark nodes can be part of the overlay itself or standalone. Each node measures its latencies to the $m$ landmarks. For node $P$, suppose that the measured distances are $\left.<l_{1}, l_{2}, \ldots, l_{\mathrm{n}}\right\rangle$. We then position node $P$ in an $m$-dimension Cartesian space using $<l_{1}, l_{2}, \ldots, l_{\mathrm{n}}>$ as its coordinates. We call this Cartesian space, the landmark space. The intuition behind doing this is that nodes that are close to each other have similar landmark measurements, and are close to each other in the landmark space. We use the Landmark Clustering technique to re-rank all the nodes in the above both lists maintained by the peer, and then update its neighborhood by selecting the first $k_{l}$ best nodes as neighbors, here $k_{l}$ is the original number of the peer's local neighbors. The aim we do this is to guarantee that all the local neighbors of a peer are really "local" both in physical proximity and in semantic proximity. 
Take the dominant resource-locating application as an example, the SplitProber algorithm makes discriminative use of nodes and probes the overlay topology by splitting the search process into two distinct sub-processes elaborated as follows.

1. Local Probing: Due to the above two kinds of proximities, a majority of queries issued by a peer are expected to be answered successfully by or through its local neighbors. Thus the peer can forward the incoming queries to all its local neighbors using scoped-flooding with a much smaller TTL. If applied with the NoN-Lookahead local indexing mechanism (addressed later), a TTL value of 2 will results in 4 hops away from the peer, which will cover a much large fraction of the overlay due to the power-law degree distributions and the smallworld-like structure.

2. Global Probing: To limit the explosive duplicate messages and heavy traffic load, we prefer random walks rather than flooding when probing long-range global neighbors. But much differently, we use a strategy, called Intentional Walks [20], to relay queries along global links towards the specified destination, say $D$. On getting close to $D$, the walks end and we then resort to Local Probing to complete the remaining search processes for the reasons addressed above.

Optionally, we also propose an optimization mechanism below to improve the performance of the SplitProber algorithm.

NoN-Lookahead local indexing. With the above procedure, when we choose a neighbor closest to the destination we do not know if it has a neighbor to take us closer to the destination. As a remedy, the clients (or just some high-degree ones) can be modified to locally keep index of the files stored by their friends, in a 2-level lookahead, their Neighbors-of-Neighbors.

\section{Evaluation Methodology}

In this section, we use simulations to evaluate SplitProber and compare its performance to three other well-known search algorithms used in unstructured overlays. Thus our simulations refer to the following four models:

1. FLOOD: Search using TTL-based flooding over the standard Gnutella topologies. This represents the classical Gnutella protocol.

2. $R W$ : Search using random walks. This represents the recommended search techniques suggested by Lv et al [15].

3. DS: Search using high-degree seeking strategies, suggested by Adamic et al [11]. In this model, queries are flooded only between high-degree nodes.

4. SplitProber: Search using our proposed SplitProber algorithm over the desirable overlays with power-law degree distributions and small-world-like structure, according the model we develop in Section 3.

We consider a system model where peers are organized in an overlay network. Each peer has a set of neighbors with which it communicates by message passing. Links are directed: a peer $P$ may have another peer $P^{\prime}$ as neighbor without $P^{\prime}$ considering $P$ as its neighbor. Traffic can however flow in both directions on the links. We use a Gnutella overlay with a $\tau=2.1$ power-law out-degree distribution and a simple 
cutoff at $m \sim N^{1 / \tau}$ to validate our analytical results. In the simulations, 100 500 unique files with varying popularity are introduced into the system. Each file has multiple copies stored at different locations chosen at random. The number of copies of a file is proportional to their popularity. The count of file copies is assumed to follow Zipf distribution with 2,000 4,000 copies for the most popular file and 40 80 copies for the least popular file. The queries that search for these files are also initiated at random hosts on the overlay topology. Again the number of queries for a file is assumed to be proportional to its popularity.

To make a comprehensive comparison with the other three algorithms and models, we focus on the following three aspects of performance metrics. These metrics, though simple, reflect the fundamental properties of search algorithms.

1. Search performance: a local gain perceived directly by a user of a system, measured along multiple dimensions such as search time, success rate of search, the cumulative nodes found, and the number of hops needed.

2. Load aspects: overhead of an algorithm, measured by the metrics like the average number of messages per node and the percentage of duplicate messages.

3. Utilization of the underlying network: a global property that is of interest to network service providers, measured by the metrics like the physical distance (latency) to the search results, and the mean stress, one of the most common definitions of the traffic load in overlay network [21].

Each experiment is repeated multiple times with different seeds to remove any bias in random number generation that is used in multiple stages of simulation.

\section{Simulation Results}

In this section, we present and analyze the results of the experimental evaluation of SplitProber. We first start by studying the overall impact of SplitProber on the search performance. We then analyze the traffic load to examine the overhead of SplitProber compared with the other three models. Finally, we evaluate the performance gains of SplitProber in the utilization of underlying network.

Fig.1(a) shows the search success rate as a function of the number of hops. The results clearly indicate that, with our proposed SplitProber algorithm, we achieve higher success rate of search even within the initial two hops, especially when the optional NoN-Lookahead local indexing mechanism is applied. The results of Fig.1(b) show that SplitProber can explore the overlay network with a search time almost equal to that of the flooding strategy, the recognized fastest strategy with regard to search in unstructured overlays. This performance gain can be explained by Fig.1(c), where SplitProber can find much more unique nodes (only a little fewer than that of FLOOD) within the same hops than those of DS and RW. To examine the role of the proximity factor $\delta$ we introduced in the SplitProber design, we plot the average number of steps for a successful query as a function of $\delta$ in Fig.1(d), the results in which show that the presence of the proximity factor with a proper value (for example, here $\delta=0.8$ ) will contribute greatly to speeding up the search process, and that it is also more beneficial to exploit the high-degree neighbors with a probability than in a simple determinate way used by DS. 

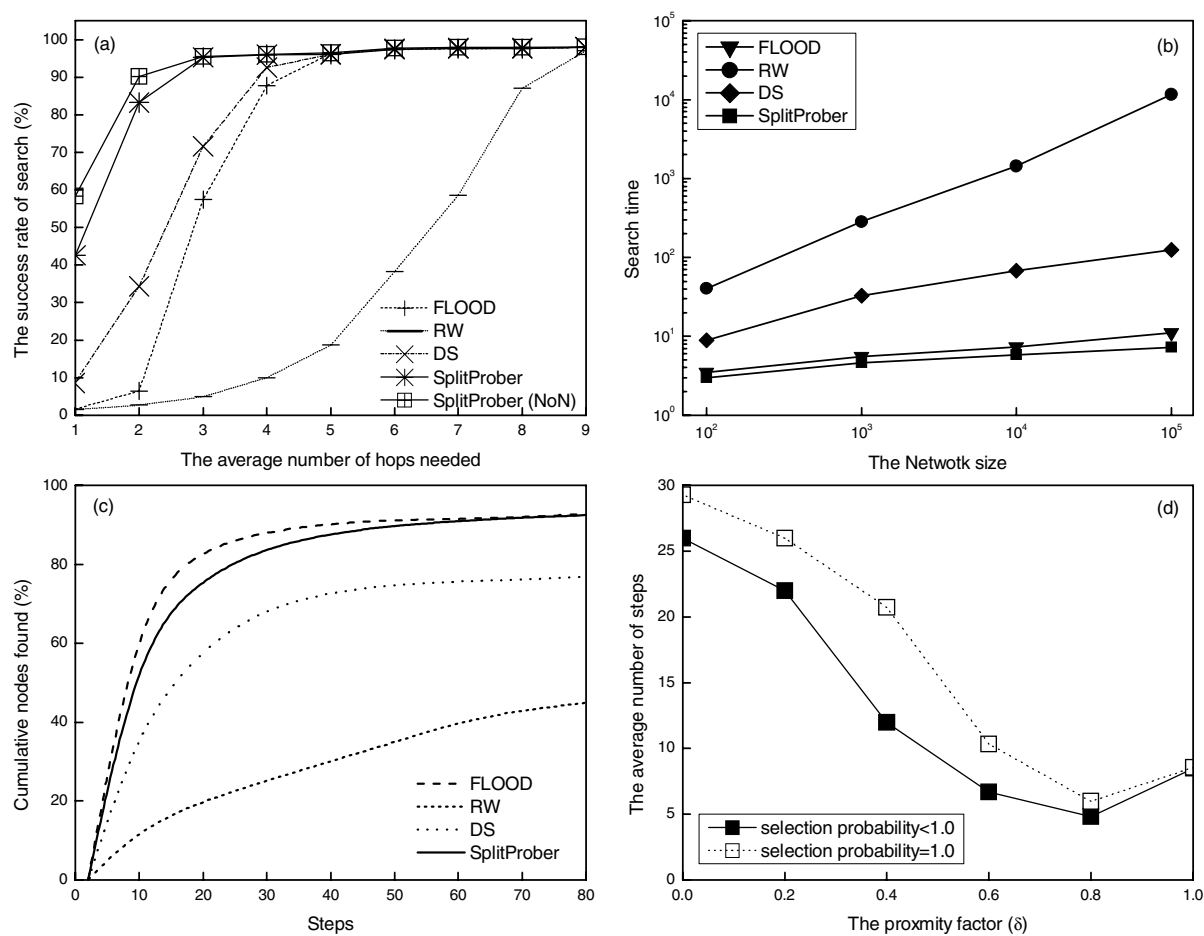

Fig. 1. The comparisons of search performance. (a) The success rate of search as a function of the number of hops. (b) Scaling of the average node-to-node search cost. Here the power-law exponent $\tau=2.1$. (c) Cumulative distribution of nodes seen vs the number of steps. (d) The average number of steps for a successful query as a function of the proximity factor, when selecting high-degree neighbors determinately or with a probability addressed in Section 3.
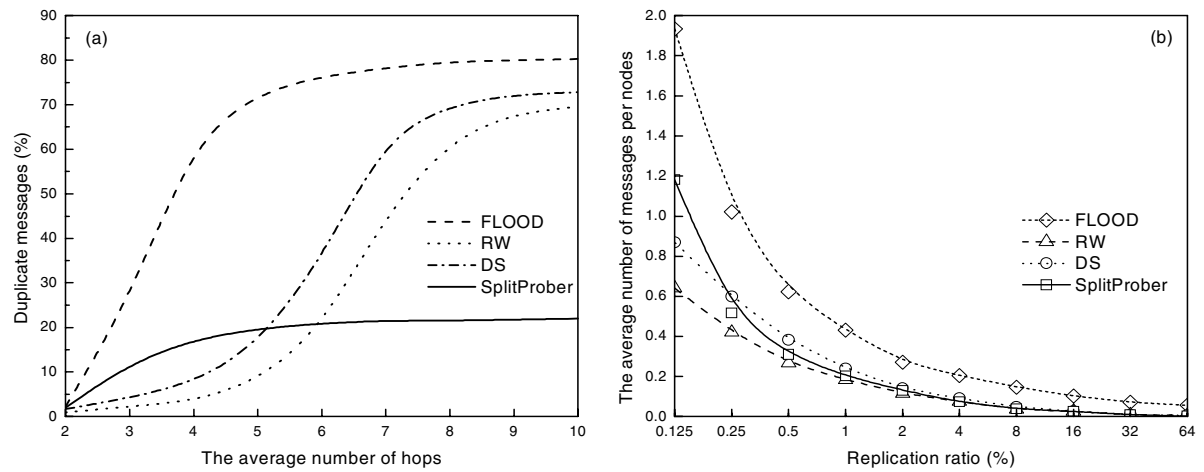

Fig. 2. The comparisons of the traffic load generated by different algorithms. (a) The percentage of duplicate messages as a function of the average number of hops traveled. (b) The average load on per node as a function of the file replication ratio. 

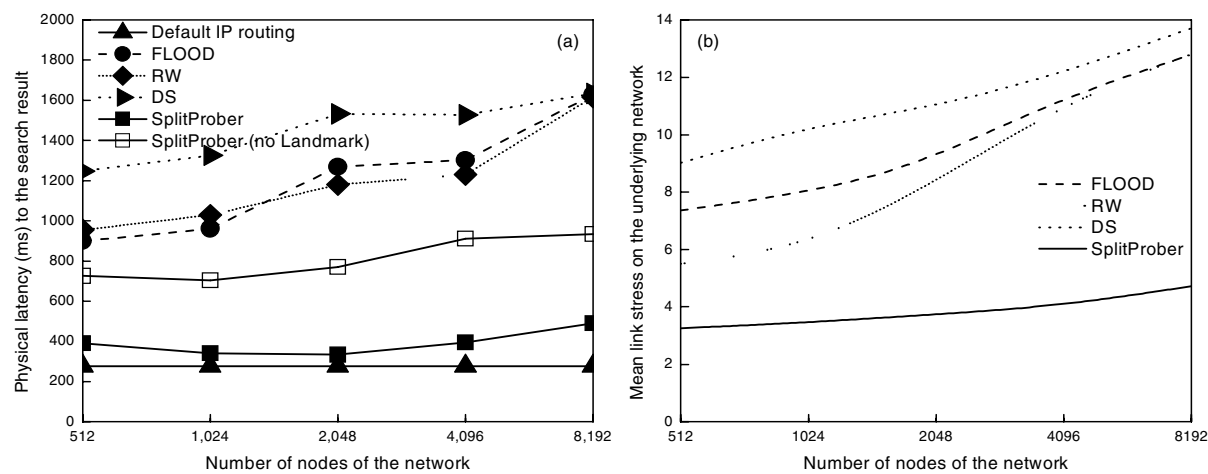

Fig. 3. The comparisons of different algorithms in the performance aspects of the utilization of underlying network. (a) The average physical distance (latency) to the search result as a function of the network size. (b) The mapping of traffic load of the overlay topology on the underlying physical links, measured by the mean link stress on the underlying network as a function of the network size.

Fig.2 plots the simulation results of traffic load. We can see from Fig.2(a) and Fig.2(b) that, SplitProber produces not only a much smaller faction of duplicate messages in the network but also fewer messages on per node, which means that the algorithm can generate lower traffic load and then distribute it more evenly across the network, with as few duplicate messages as possible.

As for the aspect of the network utilization, we can see from Fig.3 that our algorithm can make better use of the knowledge of underlying network, by dynamically optimizing the neighborhood quality to reduce the distance to search result (see Fig.3(a)), and by mapping more logical links to local physical links (see Fig.3(b)). These results further verify the significant performance gains of our proposed SplitProber algorithm.

\section{Conclusions and Future Work}

The unstructured overlay networks, while elegant from a theoretical perspective, have some serious disadvantages. First, due to knowing very little about the nature of the network topology, the search algorithms operating on these networks result in fatal scaling problems. Second, these systems rely on application-level routing, which may be inefficient with respect to network delays and bandwidth consumption. To challenge these situations, a novel local search algorithm special for these overlays, called SplitProber, is proposed in this paper. The main idea of SplitProber is to turn the power-law degree distributions in these networks into an advantage and make discriminative use of neighbor nodes according to their different roles in the network. We first develop a provably reasonable model to construct desirable overlays with power-law degree distributions and small-world-like structure. Based on this model, SplitProber probes these networks by splitting the search process into two distinct sub-processes: Local Probing and Global Probing, using two kinds of modified search strategies respectively and several optional optimization mechanisms. The simulation results form extensive experiments justify significant performance gains of SplitProber, compared with the other three well-known solutions. 
Our work in this paper mainly focuses on the search process in unstructured overlays, without considering the download process. A fact that increasing files in networks are large-sized (eg., multimedia files) [22] underscores the significance of decentralized multimedia sharing applications. Accordingly, our further work will study how to incorporate effective techniques related to large-sized (and even realtime) file download process into our algorithm and make it be more practical.

\section{References}

1. Gnutella. http://www.gnutella.wego.com

2. Clarke, I., et al. "Freenet: A Distributed Anonymous Information Storage and Retrieval System", in International Workshop on Design Issues in Anonymity and Unobservability, New York, USA, 2001

3. Ratnasamy, S., et al. "A Scalable Content-Addressable Network", in ACM SIGCOMM, San Diego, CA, USA, 2001

4. Stoica, I., et al. "Chord: A scalable peer-to-peer lookup service for Internet applications" in ACM SIGCOMM, San Diego, CA, USA, 2001

5. A. Rowstron and P. Druschel. "Pastry: Scalable, distributed object location and routing for large-scale peer-to-peer systems", International Conference on Distributed Systems Platforms (Middleware), Nov 2001.

6. Mihajlo A. Jovanovic, Fred S. Annexstein, Kenneth A. Berman. "Modeling Peer-to-Peer Network Topologies through Small-World Models and Power Laws", in Proc. of IX Telecommunications Forum Telfor, Belgrade, November 2001

7. T. Hong. "Peer-to-Peer: Harnessing the Benefits of a Disruptive Technology", edited by A. Oram (O'Reilley, ADDRESS, 2001), Chap. 14, pp. 203-241

8. D.J.Watts. "Small-worlds: The Dynamics of Networks between Order and Randomness", Princeton University Press, 1999

9. J. Kleinberg. "The small world phenomenon: an algorithmic perspective", Cornell Computer Science Technical Report 991776, 2000

10. D. J. Watts and S. H. Strogatz. "Collective dynamics of 'small-world' networks", Nature 393, 440-442, 1998

11. Adamic, L. A., Lukose R. M., Puniyani A. R., and Huberman B. A. "Search in power-law networks", http://www.parc.xerox.com/istl/groups/iea/papers/plsearch, March, 2001.

12. J. Kleinberg. "Navigation in a small world", Nature, 406, 2000

13. KaZaa Media Desktop, Sharman Networks Ltd., http://www.kazaa.com, 2001

14. B. Yang and H. Garcia-Molina. in Proc. of ICDCS 2002

15. Lv, Q., P. Cao, E. Cohen, K. Li, S. Shenker. "Search and replication in unstructured peer to peer networks", In Proc. of the 16th international conference on super-computing, Jun. 2002

16. V. Cholvi, P. Felber, E.W. Biersack, "Efficient Search in Unstructured Peer-to-Peer Networks", in European Transactions on Telecommunications, Special Issue on P2P Networking and P2P Services, Volume 15, Issue 6, 2004

17. Yang, B., P. Vinograd and H. Garcia-Molina. "Evaluating GUESS and Non-Forwarding Peer-to-Peer Search", in Proc. of ICDCS 2004

18. Clip2, http://www.clip2.com/gnutella.html, 2000

19. Zhichen $\mathrm{Xu}$, Mallik Mahalingam and Magnus Karlsson. "Turning Heterogeneity into an Advantage in Overlay Routing", in Proc. of INFOCOM'03, 2003

20. Amit R Puniyani, Rajan M Lukose, Bernardo A Huberman. "Intentional Walks on Scale Free Small Worlds", arXiv: cond-mat/0107212 v1, 11 July 2001

21. M. Ripeanu, et al, "Mapping the Gnutella Network: Properties of Large Scale Peer-to-Peer Systems and Implications for System Design," IEEE J. on Internet Computing, 2002

22. Saroiu, S., et al. "An Analysis of Internet Content Delivery Systems", In Proc. of the Fifth Symposium on Operating Systems Design and Implementation, Boston, MA, Dec. 2002 